\title{
Vivax malaria with leucopenia and thrombocytopenia: a report of 3 cases
}

\author{
Priti Khemka $^{1}$, Swapan Ray $^{2}$, Sukanta Bhattacharya ${ }^{3}$, Mallar Mukherjee ${ }^{4}$, Pawan Nandurkar ${ }^{1}$
}

Sri Lanka Journal of Child Health 2013; 42(3): 161-162

(Keywords: Plasmodium vivax; malaria; leucopenia; thrombocytopenia)

\section{Introduction}

The occurrence of thrombocytopenia during complicated falciparum malaria is well known. However, there are few reports on the occurrence of both leucopenia and thrombocytopenia with vivax malaria. We would like to report 3 cases of vivax malaria presenting with leucopenia and severe thrombocytopenia.

\section{Case reports}

Three children were admitted to our institute between July and August 2012 with complaints of fever. One of them presented with a petechial rash over both legs. None of them had mucosal bleeds. There was no other evidence of coagulopathy. None had altered sensorium, liver dysfunction or renal failure. Monoinfection with vivax malaria was established from peripheral blood smears and rapid diagnostic tests. All 3 had leucopenia and severe thrombocytopenia. All 3 patients had negative blood cultures, negative Widal tests and negative dengue serology. Other causes of thrombocytopenia were ruled out. All of them were treated with artemisin combination therapy and primaquine. None of the cases required platelet transfusions and all of them recovered completely following antimalarial therapy. The clinical and laboratory findings of the 3 cases are shown in Table 1.

Table 1: Clinical and laboratory findings of the 3 cases

\begin{tabular}{|c|c|c|c|}
\hline & Case 1 & Case 2 & Case 3 \\
\hline Age & 8 years & 4 years & 2 years \\
\hline Sex & Female & Male & Male \\
\hline Duration of fever & 8 days & 11 days & 4 days \\
\hline Hepatomegaly & Yes & Yes & No \\
\hline Splenomegaly & No & Yes & No \\
\hline Haemoglobin & $9.2 \mathrm{~g} / \mathrm{dl}$ & $6.4 \mathrm{~g} / \mathrm{dl}$ & $11.6 \mathrm{~g} / \mathrm{dl}$ \\
\hline Total leucocyte count on admission & $3.7 \times 10^{9} / 1$ & $2.8 \times 10^{9} / 1$ & $3.9 \times 10^{9} / 1$ \\
\hline Total leucocyte count on discharge & $6.3 \times 10^{9} / 1$ & $4.2 \times 10^{9} / 1$ & $6.8 \times 10^{9} / 1$ \\
\hline Platelet count on admission & $45 \times 10^{9} / 1$ & $38 \times 10^{9} / 1$ & $48 \times 10^{9} / 1$ \\
\hline Platelet count on discharge & $373 \times 10^{9} / 1$ & $181 \times 10^{9} / 1$ & $152 \times 10^{9} / 1$ \\
\hline Peripheral blood smear & P. vivax & P. vivax & P. vivax \\
\hline Rapid diagnostic test & P. vivax & P. vivax & P. vivax \\
\hline
\end{tabular}

\section{Discussion}

Falciparum malaria has traditionally been known as malignant tertian malaria and vivax malaria as benign tertian malaria. However, with the increasing number of complications being reported with vivax malaria these terms may no longer be applicable ${ }^{1}$.

\section{${ }^{1}$ Post-graduate trainee, ${ }^{2}$ Associate Professor, ${ }^{3}$ Professor, ${ }^{4}$ Senior Resident, Department of Paediatrics, Institute of Child Health, Kolkata, India}

(Received on 8 September 2012: Accepted after revision on 19 October 2012)
Complications with vivax malaria include cerebral malaria, severe anaemia, severe thrombocytopenia, hepatic dysfunction, acute renal failure, acute lung injury, acute respiratory distress syndrome and shock $^{1,2}$. Uncomplicated vivax malaria may also have derangements in blood parameters like leucopenia and thrombocytopenia ${ }^{3,4}$. Thrombocytopenia is the commonest complication of vivax malaria ${ }^{5-7}$.

In a study by Jadhav UM et al, it was found that thrombocytopenia was seen in both vivax and falciparum malaria and that platelet counts were lower in falciparum malaria ${ }^{8}$. However platelet counts could not be used to distinguish between the 2 
types of malaria ${ }^{8}$. In a study from Venezuela, thrombocytopenia was seen in more than $50 \%$ of patients with vivax malaria and around $50 \%$ of these patients with severe thrombocytopenia required platelet transfusions ${ }^{9}$. They also reported that that leucopenia was more common among patients with $P$. vivax infection than among those infected with $P$. falciparum ${ }^{4}$. Another study reported that white blood cell counts were lower in falciparum malaria than in vivax malaria ${ }^{3}$.

The pathogenesis of thrombocytopenia in vivax malaria has not been well defined. Proposed mechanisms include immune mediated lysis, oxidative stress, sequestration in spleen and diminished platelet production. Abnormalities in platelet structure and function and direct interaction between plasmodium and platelets have also been described $^{7,8}$.

Thrombocytopenia is a common but less recognized feature of acute malaria. Low platelet counts may be transient during the illness and improve upon recovery. No specific treatment is required other than treatment of the cause. Platelet infusions must be reserved for severe cases and decided on an individual case basis.

\section{References}

1. Srivastava S, Ahmad S, Shirazi N, Kumar Verma S, Puri P. Retrospective analysis of vivax malaria patients presenting to tertiary referral centre of Uttarakhand. Acta Tropica 2011; 117(2):82-5

http://dx.doi.org/10.1016/j.actatropica.2010.10.001

2. Naha K, Dasari S, Prabhu M. Spectrum of complications associated with Plasmodium vivax infection in a tertiary hospital in South-Western
India. Asian Pacific Journal of Tropical Medicine 2012; 5(1):79-82.

http://dx.doi.org/10.1016/S19957645(11)60251-4

3. McKenzie FE, Prudhomme WA, Magill AJ, Forney JR, Permpanich B, Lucas C, et al. White blood cell counts and malaria. Journal of Infectious Diseases 2005; 192(2):323-30. http://dx.doi.org/10.1086/431152

4. Rodríguez-Morales AJ, Sánchez E, Arria M, Vargas M, Piccolo C, Colina R, et al. White blood cell counts in Plasmodium vivax malaria. Journal of Infectious Diseases 2005; 192 (9):1675-6. http://dx.doi.org/10.1086/496993

5. Katira B \& Shah I. Thrombocytopenia in Plasmodium vivax infected children. Journal of Vector Borne Diseases 2006; 43: 147-9.

6. Kumar A, Shashirekha. Thrombocytopenia--an indicator of acute vivax malaria. Indian Journal of Pathology and Microbiology 2006; 49(4):505-8.

7. Bhandary N, Vikram G S, Shetty H. Thrombocytopenia in malaria: A clinical study. Biomedical Research 2011; 22 (4): 489-91.

8. Jadhav UM, Patkar VS, Kadam NN. Thrombocytopenia in malaria - Correlation with type and severity of malaria. Journal of the Association of Physicians of India 2004; 52:615-8.

9. Rodriguez-Morales AJ, Sanchez E, Vargas M, et al. Occurrence of thrombocytopenia in Plasmodium vivax malaria. Clinical Infectious Diseases 2005; 41 (1): 130-1. http://dx.doi.org/10.1086/430837 